\title{
BRST Cohomology in Classical Mechanics
}

\author{
Marc Henneaux $^{1,2, \star}$ and Claudio Teitelboim ${ }^{2,3}$ \\ ${ }^{1}$ Faculté des Sciences, Université Libre de Bruxelles, Campus Plaine C.P. 231, B-1050 Bruxelles, \\ Belgium \\ ${ }^{2}$ Centro de Estudios Científicos de Santiago, Casilla 16443, Santiago 9, Chile \\ 3 Center for Relativity, The University of Texas at Austin, Austin, TX 78712, USA
}

\begin{abstract}
The classical (non-quantum) cohomology of the Becchi-RouetStora-Tyutin (BRST) symmetry in phase space is defined and worked out. No group action for the gauge transformations is assumed. The results cover, therefore, the general case of an "open algebra" and are valid "off-shell." Each cohomology class contains all BRST invariant functions with fixed ghost number (an integer) which differ from each other by a BRST variation. If the ghost number is negative there is only the trivial class whose elements are equivalent to zero. If the ghost number is positive or zero there is a bijective correspondence between the BRST classes and those of the exterior derivative along the gauge orbits. These gauge orbits lie in the phase space surface on which the gauge generators are constrained to vanish. The BRST invariant functions of ghost number $p$ are then related to closed $p$-forms along the orbits. The addition of a BRST variation corresponds to the addition of an exact form. Some comments about the quantum case are included. The physical meaning of the classes with ghost number greater than zero is not discussed.
\end{abstract}

\section{Introduction}

The concept of ghost has steadily emerged as one of central importance in the study of the dynamics of physical systems endowed with a local geometric structure or, as one says, of gauge systems.

Ghosts were first encountered in quantum field theory as fields with the "wrong" relation between spin and statistics which were necessary in addition to those which appeared in the correspondent classical problem [1]. The ghost fields ensured that the theory would be unitary. This they did through contributions to virtual processes only, thus avoiding conflict with the spin-statistics theorem.

After the advent of the Faddeev-Popov measure for summing over classes of equivalence of gauge field histories [2], the ghosts were regarded as just an artifact leading to a useful representation of that measure. However they were restored to a somewhat more respectable role when it was realized that in more complicated

\footnotetext{
* Chercheur qualifié du Fonds National de la Recherche Scientifique (Belgium)
} 
theories it was necessary to include self interactions of the ghosts in order to achieve unitarity and this could not be fitted in the Faddeev-Popov scheme.

However, what raised the ghost to a prominent role was the discovery of the Becchi-Rouet-Stora-Tyutin (BRST) symmetry [3, 4]. Since this symmetry mixes the ghosts with the other fields it became clear that one should regard all the fields, including the ghosts, as different "components" of a single geometrical object. This point of view emerged as the logical development of the idea of gauge invariance.

The need for ghosts and the symmetry that reveals their profound importance were first established in quantum mechanics. It was only afterwards realized that they have a natural and necessary place within classical mechanics as well. Indeed, the BRST symmetry could have been discovered in the last century within a strictly classical context by mathematicians dealing with the geometry of phase space, had they only been willing to extend their analysis to Grassmann variables.

Having said this, it should be emphasized that we are not advocating that ghosts should have direct physical meaning within classical mechanics. It is nevertheless extremely useful to be able to discuss the BRST symmetry classically. One can then bring in this concept as a powerful tool in the actual construction of the quantum theory, rather than of having to wait to have the quantum theory to discuss it.

The central property of the BRST symmetry is its nilpotency: if applied twice it gives zero. This, of course, makes one think that the symmetry should have a topological meaning.

This paper is devoted to making a step in that direction. We have studied the cohomology of the BRST symmetry within the context of classical mechanics and have found that it can be related to the cohomology of the exterior derivative along the phase space gauge orbits. This relationship will be made precise below, but we emphasize already here that we do not need to assume group property for the gauge transformations. Our results hold for gauge transformations which only close modulo the equations of motion, that is for a gauge algebra that only closes "on-shell."

The plan of the paper is the following: Section II reviews the BRST symmetry in phase space. It begins with a brief discussion of gauge invariance and constraints and then it goes on to treat the extension of phase space to include classical ghosts. Next, the general properties of the BRST operator are presented. It is emphasized that it exists and it is identically ("off-shell") nilpotent even when the gauge transformations do not form a group. It is also stressed that the BRST operator does not need any gauge fixing to be written down, and consequently is independent of any gauge condition that one might eventually need to bring in for other reasons. Lastly, it is pointed out that the BRST construction reveals that different representations of the constraint surface can be thought of as being obtained from each other by a canonical transformation in the extended phase space ("canonical covariance"). In this sense, for a given gauge theory the BRST generator in the extended phase space is unique and so is the cohomology based on it.

The notion of the classical BRST cohomology is introduced in Sect. III. It begins with the observation that the nilpotency of the BRST operator induces a cohomology through the Poisson bracket. It is next recalled that BRST invariants 
with zero ghost number are equivalent to gauge invariant functions. Next, we go on to discuss BRST invariance with ghost number different from zero. The theorem that contains the new results presented in this paper is then outlined and explained in brief.

Section IV introduces a purely geometrical concept that will then be associated in Sect. V with the BRST symmetry. It is the idea of the exterior derivative along orbits generated by the constraints within the constraint surface. This exterior derivative possesses a cohomology which is not identical to the ordinary De Rham cohomology of phase space, or of a given orbit.

Finally, the BRST cohomology with non-zero ghost number is discussed in Sect. V. It is shown that when the ghost number is negative the cohomology is trivial, and when it is positive, the cohomology is isomorphic to that of the exterior derivative treated in Sect. IV.

Also addressed, and answered in the negative, in this section is the question of whether the BRST cohomology explicitly sees all the structure there is. Lastly, the classical and quantum cohomologies are compared and contrasted.

\section{BRST Symmetry in Phase Space}

\section{Gauge Invariance and Constraints}

One says that a dynamical system with phase space coordinates $(q, p)$ possesses a gauge invariance or, in brief, is a gauge system, if the equations of motion do not determine uniquely $q(t)$ and $p(t)$ once $q\left(t_{0}\right)$ and $p\left(t_{0}\right)$ are given for an initial time $t$. By definition, one then says that all the $q(t), p(t)$ which spring from the same $q\left(t_{0}\right), p\left(t_{0}\right)$ are physically indistinguishable and are related to each other by a gauge transformation. The gauge transformation turns out to be a canonical transformation whose generators will be denoted $G_{a}(q, p)$. It has been shown by Dirac [5] that for a gauge system the admissible states lie within the hypersurface in phase space on which the gauge generators take the numerical value zero,

$$
G_{a}(q, p)=0, \quad a=1, \ldots, m .
$$

These equations will be called the constraint equations, and for that reason the generators themselves will be sometimes called by abuse of language, "the constraints." The surface defined by (II.1.1) will be called the constraint surface.

The fact that the commutation of two gauge transformations gives ("on-shell") a new gauge transformation is reflected in the existence of a set of structure functions $C_{b c}^{a}(q, p)$ such that one has the Poisson bracket relation,

$$
\left[G_{a}, G_{b}\right]=C_{a b}^{c}(q, p) G_{c} .
$$

When the functions $C_{a b}^{c}$ turn out to be constant, the gauge transformations generated by the $G_{a}$ 's close everywhere in phase space. Otherwise, they only close on the constraint surface defined by (II.1.1).

The property of the constraints that the Poisson bracket of any two of them is a linear combination of the constraints themselves is called the "first class property" 
and the constraints are called "first class". ${ }^{1}$ Geometrically (II.1.2) says that the motions in phase space generated by $G_{a}$ are tangent to the constraint surface (which is thus a coisotropic surface in symplectic terminology).

Equations (II.1.2) were heavily exploited by Dirac in his analysis which established the foundation of the theory of constrained systems. However, it was only recently realized $[7,8]$ that by taking repeated antisymmetrized Poisson brackets of (II.1.2) with the generators $G_{a}$, new "higher order" structure functions are generated which obey a remarkable set of identities, thus originating an additional interesting structure. This structure which is obviously completely contained in classical mechanics turns out to be at the heart of the BRST symmetry. More precisely, the higher order structure functions may be used to construct the BRST generator. The identities in question become then equivalent to the fundamental properties of the BRST symmetry, among them the crucial nilpotency. The BRST construction thus captures succinctly all the gauge structure as we now pass to summarize.

\section{Classical Ghosts}

One enlarges the original phase space of the $q$ 's and the $p$ 's by introducing canonically conjugate pairs $\left(\eta^{a}, \mathscr{P}_{a}\right)$ of Grassmann parity opposite to the one of the associated constraints ${ }^{2}$

$$
\left[\mathscr{P}_{a}, \eta^{b}\right]=-\delta_{a}^{b}
$$

We allow for the possibility that some of the original $(q, p)$ may be commuting and the other anticommuting. The Poisson bracket used throughout is therefore the appropriate graded bracket.

The extra variables $\left(\eta^{a}, \mathscr{P}_{a}\right)$ introduced as additional canonical coordinates in the classical mechanics of a gauge system are called ghosts. They may be initially thought of as a useful book-keeping device for concisely describing the Poisson bracket structure of the system. However this narrow view lends itself to rapid change once one realizes that the structure in question is equivalent to the existence of a symmetry in the enlarged phase space, which mixes the ghosts with the original variables. This is summarized next and is what makes it clear that the most appropriate arena for describing the classical dynamics of a gauge system is

\footnotetext{
${ }^{1}$ Second class constraints can always (at least locally) be viewed as gauge-fixing conditions of a larger, equivalent system with a gauge invariance, to which one can apply the BRST construction [6]. One can thus say that (at least locally) first class constraints cover the general case

${ }^{2}$ In order to simplify technical considerations, we assume the constraints to be independent ("irreducible case"). To be more precise, the Jacobian matrix $\left(\partial G_{a} / \partial q^{i}, \partial G_{a} / \partial p_{i}\right)$ is of "maximal rank" everywhere on the constraint surface, i.e., locally, one can take $G_{a}$ as first $m$ coordinates in phase space $\left[0\right.$ is a regular value of the mapping defined by $\left.G_{a}(q, p)\right]$. This assures, under suitable regularity conditions which will also be assumed, that
}

$$
F^{a_{1} \ldots a_{p}} G_{a_{p}}=0 \Leftrightarrow F^{a_{1} \ldots a_{p}}=K^{a_{1} \ldots a_{p} a_{p+1}} G_{a_{p+1}}(-)^{\varepsilon_{a_{p+1}}},
$$

where $F$ and $K$ are "antisymmetric" in their indices (see [8] for a proof which does not rely on a group action). The reducible case possesses also a nilpotent BRST charge $[9,8]$ but will not concern us here 
not the original phase space of the $q$ 's and the $p$ 's, but rather the extended phase space which also includes the ghosts. From this point of view, the BRST symmetry could have been discovered even before the advent of quantum mechanics and also independently of the existence of Lie groups, since the analysis does not need that the $C_{b c}^{a}$ be constants.

\section{Existence of the BRST Generator}

The way in which gauge invariance is described in the extended phase space is contained in the following

Theorem 1. To any first-class system, one can associate a Grassmann-odd BRST generator $\Omega$ characterized by

$$
\begin{gathered}
g h \Omega=1, \\
\Omega=\eta^{a} G_{a} \\
+ \text { terms which vanish with the ghost momenta, } \\
{[\Omega, \Omega]=0 \quad \text { ("nilpotency"), }} \\
\Omega=\Omega^{*} .
\end{gathered}
$$

The ghost number appearing in (II.3.1a) is defined by stating that

$$
g h \eta^{b}=-g h \mathscr{P}_{a}=1, \quad g h q^{i}=g h p_{i}=0,
$$

and the assumption that the ghost number of a product is the sum of the ghost number of the factors.

The construction of $\Omega$ was given for the first time in the remarkable work [7] and generically involves multi-ghost terms. A complete, explicit proof of the theorem may be found in [8].

An important feature of $\Omega$ is that (II.3.1c) holds independently of the equations of motion. To achieve this "off-shell nilpotency," it is necessary to work within the canonical formalism, since it has been proved in [10] that nilpotency cannot hold off-shell in the Lagrangian approach when the "algebra" of the gauge transformations only close on-shell. In that sense, one can say that the conjugate momenta are the "auxiliary fields" necessary to close the BRST algebra (II.3.1c) off-shell.

Another remarkable feature of the Hamiltonian BRST charge is its manifest gauge independence. No gauge condition is ever needed to construct it, and $\Omega$ possesses therefore a clear intrinsic meaning.

\section{Uniqueness of the BRST Generator}

The reformulation of the idea of gauge invariance in terms of the BRST generator has an immediate important pay-off. In the original phase space of the $p$ 's and the $q$ 's, one may describe the same constrained surface in terms of a set of constraints $G_{a}(q, p)$, or in terms of another set $\bar{G}_{a}$ related to the first one by

$$
\bar{G}_{a}=a_{a}^{b}(q, p) G_{b}, \quad \operatorname{det} a_{a}^{b} \neq 0 .
$$

Here $a_{a}^{b}(q, p)$ is a general element of $G L(n, R)$ which varies from point to point in phase space. 
The two descriptions are equivalent in the sense that they describe the same constraint surface. However, the structure functions (of all orders) are quite different in both cases and their relationship is awkward. It may happen for example that for the $G_{a}$ 's one will have $C^{a}{ }_{b c}$ independent of $q$ and $p$ and therefore a Lie group structure, while that will not be the case for the $\bar{G}_{a}(q, p)$. Thus a complete understanding of the equivalence goes beyond the simple statement that both choices of generators define the same constraint surface. The BRST construction solves that problem through the following $[8,11]$.

Theorem 2. Let $\Omega$ and $\bar{\Omega}$ be two BRST charges associated with the same constraint surface. Then, $\Omega$ and $\bar{\Omega}$ are related by a canonical transformation in the extended phase space.

This theorem was actually only proved in [8] for invertible linear transformations which are in the connected component of the identity, i.e., which have positive determinant. The general case with both positive and negative determinants is easily covered by observing that the particular matrix $a_{b}^{a}=\operatorname{diag}(-1,1, \ldots, 1)$, which possesses negative determinant, can be generated by the ghost canonical transformation $\eta^{1} \rightarrow-\eta^{1}, \mathscr{P}_{1} \rightarrow-\mathscr{P}_{1}, \eta^{a} \rightarrow \eta^{a}, \mathscr{P}_{a} \rightarrow \mathscr{P}_{a}(a \geqq 2)$.

One may also describe Theorem 2, as containing the "canonical covariance" of the whole structure of first class constrained systems, which is otherwise obscure without the ghosts. Hence, one can say that the ghost serve the classical purpose of making manifest the canonical covariance of the structure of "coisotropic" surfaces, and, it is for this reason that it is fair to believe that the BRST construction could have been invented long ago.

In the sense of Theorem 2, one associates a unique BRST generator in the extended phase space to a given gauge theory.

\section{Classical BRST Cohomology}

\section{Nilpotency of BRST Symmetry Induces Cohomology Through Poisson Brackets}

The "off-shell" nilpotency of the BRST transformation enables one to introduce the classical (non-quantum) BRST cohomology for any gauge theory. Indeed, it follows from (II.3.1c) and the Jacobi identity for the graded Poisson brackets that

$$
[[A, \Omega], \Omega]=0
$$

for any function $A(q, p, \eta, \mathscr{P})$ on the extended phase space. Hence, one can define BRST-closed functions as functions which are BRST invariant,

$$
[A, \Omega]=0,
$$

while BRST-exact functions are given by

$$
A=[K, \Omega]
$$

for some $K$, and are clearly BRST-closed.

As a result of Theorem 2, the classical BRST cohomology only depends on the first-class constraint surface defining the dynamical system under consideration, and not on how one represents this surface by implicit equations $G_{a}(q, p)=0$, or on 
how one removes, at each stage, the ambiguity in the structure functions entering the construction of $\Omega$. Furthermore, the BRST cohomology is also gauge invariant. This is because $\Omega$ is gauge independent.

Because the BRST charge possesses ghost number +1 , one can study cohomology classes (III.1.2), (III.1.3) with given ghost number. We thus define

$$
\left(\frac{\operatorname{Ker} \Omega}{\operatorname{Im} \Omega}\right)_{\text {classical }}^{g}
$$

as the set of equivalence classes of BRST-closed functions modulo BRST-exact functions, with definite ghost number $g$.

\section{BRST Invariants with Ghost Number Zero Equivalent to Gauge Invariant Functions}

The classical cohomology of $\Omega$ with $g=0$ has been completely characterized in [8] through the following,

\section{Theorem 3.}

$$
\left(\frac{\operatorname{Ker} \Omega}{\operatorname{Im} \Omega}\right)_{\text {classical }}^{0}
$$

is isomorphic to the set of equivalence classes of gauge invariant "observables" $A_{0}(q, p)$, where two gauge invariant observables $A_{0}$ and $A_{0}^{\prime}$ are identified if they take the same values on the constraint surface (weak equality: $A_{0} \sim A_{0}^{\prime}$ iff $A_{0}=A_{0}^{\prime}$ $\left.+k^{a} G_{a}\right)$.

3. BRST Invariants with Ghost Number Different from Zero.

Theorem in Brief

Since the BRST cohomology with vanishing ghost number can be related to phase space functions which possess a direct physical meaning, it is natural to ask whether BRST cohomological classes with non-vanishing ghost number also have an interpretation in terms of familiar phase space objects.

The main result of this paper is to connect BRST invariant functions with closed forms along the orbits generated in phase space by the gauge transformations. More precisely, we establish the following,

\section{Theorem 4.}

$$
\left(\frac{\operatorname{Ker} \Omega}{\operatorname{Im} \Omega}\right)_{\text {classical }}^{g}=\left\{\begin{array}{cc}
0 & g<0 \\
\left(\frac{\operatorname{Ker} d}{\operatorname{Im} d}\right)^{g} & g \geqq 0
\end{array} .\right.
$$

Here, $d$ generalizes the exterior derivative operator acting along the gauge orbits generated by the constraint functions $G_{a}$ on the constraint surface. This operator will be defined more precisely in the next section. It acts on forms which are defined throughout phase space, but differs from the phase space exterior derivative operator in that it only takes derivatives along the gauge orbits, and not 
along directions orthogonal to them. So, this operator $d$ measures the variations of the forms under gauge transformations only, and remains insensitive to how the forms vary in transverse directions.

Zero forms which are closed are constant along the gauge orbits and thus, are just gauge invariant functions. Formula (III.3.1b) reduces therefore in this case to (II.2.1).

Although the above theorem provides a phase space geometrical interpretation of the BRST cohomology in the case of non-vanishing ghost number, the physical meaning and use of the cohomological classes with $p>0$ is still to be uncovered. We just point out that one may get non-trivial gauge invariant functions from non-trivial closed $p$-forms, by integrating them along non-trivial closed $p$-subsurfaces of the gauge orbits. Also, one expects cohomological classes with $p=1$ to be related to anomalies, as in the closed algebra case $[12,13]$.

\section{Exterior Derivative Along Gauge Orbits}

\section{Gauge Orbits}

Off the constraint surface, the canonical transformations generated by the constraint functions $G_{a}$ do not close - i.e., do not form a $m$-dimensional manifold when the first order structure functions $C^{a}{ }_{b c}(q, p)$ are not constant. This is because in that case the associated vector fields $X_{a}$, defined by

$$
X_{a} f \equiv \partial_{a} f \equiv\left[f, G_{a}\right], \quad a=1, \ldots, m
$$

for any function $f$, do not possess Lie brackets which are linear combinations of the vectors $X_{a}$ themselves,

$$
\left[X_{a}, X_{b}\right] \equiv X_{a} X_{b}-(-)^{\varepsilon_{a} \varepsilon_{b}} X_{b} X_{b} \neq X_{c} f_{a b}^{c} .
$$

Here, $\varepsilon_{a}$ is the "Grassmann parity" of the constraint function $G_{a}$, which indicates whether it is even $\left(\varepsilon_{a}=0\right)$ or odd $\left(\varepsilon_{a}=1\right)$. It determines the commutation/anticommutation properties of $G_{a}$ as

$$
G_{a} G_{b}=G_{b} G_{a}(-)^{\varepsilon_{b} \varepsilon_{a}} .
$$

On the constraint surface, however, one finds

$$
\left[\mathrm{X}_{a}, \mathrm{X}_{b}\right] \approx(-)^{\varepsilon_{c}+\varepsilon_{c}\left(\varepsilon_{a}+\varepsilon_{b}\right)} X_{c} C^{c}{ }_{b a},
$$

so that the vector fields $X_{a}$ generate $m$-dimensional submanifolds. These are the orbits of the gauge transformations, which we will call "gauge orbits" (in phase space) hereafter.

The constraint surface is thus partitioned into disjoint $m$-dimensional orbits. The so-called reduced phase space is the quotient space of the constraint surface by these orbits [14].

At any point on a gauge orbit, one has a tangent frame $\left\{X_{a}\right\}$, which is everywhere regular because we have assumed that the constraints are irreducible. Accordingly, a definite representation of the constraint surface by means of 


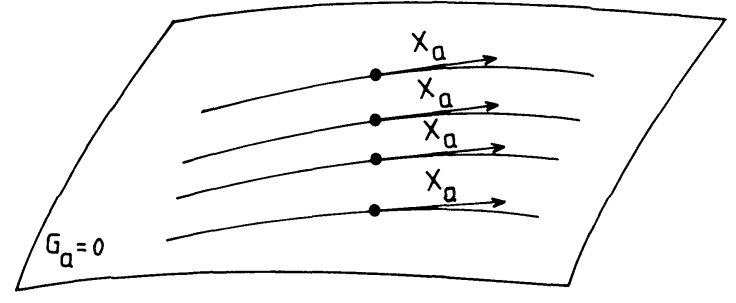

Fig. 1. The constraint hypersurface $G_{a}=0$ is a $2 n-m$ dimensional manifold embedded in phase space (assumed to be of dimension $2 n$ ). It is sliced by the $m$-dimensional gauge orbits generated by the vector fields $X_{a}$. The $m$ dual 1 -forms "tangent" to the gauge orbits are denoted by $\omega^{a},\left\langle\omega^{a}, X_{b}\right\rangle$ $=\delta_{b}^{a}$. Generalized $p$-forms considered here are defined throughout phase space, but are polynomials in the $\omega^{a}$ only (and not arbitrary polynomials in $d q^{i}, d p_{i}$ ). Two such forms which coincide on the constraint surface are identified. The exterior derivative operator $d$ along the gauge orbits acts on these forms and takes only (antisymmetrized) derivatives along the gauge orbits, not along transverse directions

implicit equations $G_{a}=0$, where 0 is a regular value of $G_{a}$, provides us with a definite tangent frame to the gauge orbits (see Fig. 1).

\section{Exterior Derivative}

On each of the gauge orbits, viewed as a $m$-dimensional manifold, one can define everywhere a dual frame $\omega^{a}$ obeying

$$
\left\langle\omega^{a}, X_{b}\right\rangle=\delta_{b}^{a}, \quad a, b=1, \ldots, m .
$$

The exterior derivative operator $d$ along a single orbit is such that

$$
d \omega^{a}=\frac{1}{2}(-)^{\varepsilon_{a}+\varepsilon_{b}} \omega^{b} \omega^{c} C_{c b}^{a}
$$

for $\omega^{a}$,

$$
d F=\partial_{a} F \omega^{a}
$$

for a scalar, and

$$
d A B=A d B+(-)^{\varepsilon_{B}}(d A) B
$$

for arbitrary forms $\left(\varepsilon_{B}\right.$ is the total Grassmann parity of the $p$-form $B$ obtained by giving Grassmann parity $\varepsilon_{a}+1$ to $\omega^{a}$; in the pure bosonic case, $\varepsilon_{B}$ coincides with the rank, see appendix). One easily checks that $d^{2}=0$ using the identity obeyed by the first order structure functions [8]. This is, of course, because $d$ in (IV.2.2-4) is just the standard exterior derivative operator acting on a given $m$-dimensional (super) manifold.

Now, instead of considering $p$-form fields defined on a given orbit, one can consider "generalized $p$-form fields along the orbits." These are simply obtained by assuming, in the expansion of $A$,

$$
A=\omega^{a_{1}} \ldots \omega^{a_{p}} A_{a_{p} \ldots a_{1}}
$$

that the coefficients $A_{a_{p} \ldots a_{1}}$ are not just functions on a given orbit, but rather, are functions defined everywhere on the constraint surface. 
Equivalently, the coefficients can be viewed as functions of the phase space coordinates $q, p$, provided one identifies two $A$ 's which are equal on the constraint surface

$$
\begin{aligned}
& A=\omega^{a_{1}} \ldots \omega^{a_{p}} A_{a_{p} \ldots a_{1}}(q, p), \\
& \mathrm{A} \sim \mathrm{A}^{\prime} \Leftrightarrow A_{a_{p} \ldots a_{1}}^{\prime}=A_{a_{p} \ldots a_{1}}+k_{a_{p} \ldots a_{1}}^{b} G_{b} .
\end{aligned}
$$

The objects (IV.2.5), with the identification (IV.2.6) will be the basic objects of our study and will be simply called generalized $p$-forms. They will be related in the next section to BRST invariant functions.

The generalized $p$-forms (IV.2.5) differ from phase space $p$-forms in that they are not arbitrary polynomials in the $2 n$ forms $d q^{i}, d p_{i}$, but rather, they only involve the smaller set of 1-forms $\omega^{a}$ tangent to the gauge orbits. They differ from $p$-forms along a given gauge orbit in that the coefficients of $\omega^{a_{1}} \ldots \omega^{a_{p}}$ in the expansion (II.2.5) are not functions defined on that given orbit, but rather, belong to the larger class of phase space functions. In that sense, one can say that the generalized $p$-forms (IV.2.5), (IV.2.6) lie somewhere in between phase space $p$-forms and $p$-forms along a given orbit.

By using the rules (IV.2.2-4) one can define the exterior derivative $d A$ of a generalized form $A$, which is also a generalized form, since only derivatives along $X_{a}$ are taken in (IV.2.3). It is easy to see that $d^{2} \approx 0$ and that the first class property of the constraints implies

$$
A \sim A^{\prime} \Rightarrow d A \sim d A^{\prime} .
$$

Of special interest are closed $p$-forms, obeying

$$
d A \approx 0 .
$$

Since $d^{2} \approx 0$, exact $p$-forms

$$
A \approx d B
$$

are closed, and one can define the cohomology of $d$ modulo the constraints. We denote the corresponding space of equivalence classes by

$$
\left(\frac{\operatorname{Ker} d}{\operatorname{Im} d}\right)^{p} \text {. }
$$

For $p=0$,(IV.2.9) yields the gauge invariant functions, with the identification of two "observables" which coincide on the constraint surface. For $p>0$, one can also associate gauge invariants to (IV.2.9), by integrating closed $p$-forms along closed $p$-surfaces lying on the gauge orbits (in the even case).

\section{Gauge Orbit Cohomology not Identical to Ordinary De Rham Cohomology}

One can call the cohomology which we have just defined the cohomology of the gauge orbits with coefficients in the space of functions of the constraint surface. Equivalently it may be called the weak cohomology of the gauge orbits with coefficients in the space of phase space functions. 
It is important to realize that this cohomology is neither equal to the ordinary De Rham cohomology of phase space, nor equal to the ordinary De Rham cohomology of a given orbit. This is because the $p$-forms which we are considering, are neither phase space $p$-forms, nor $p$-forms along a given orbit. The ingredient of the cohomology of $d$, is the whole family of gauge orbits, filling the constraint surface ${ }^{3}$.

\section{BRST Cohomology with Non-Zero Ghost Number}

We are now in a position to relate the classical BRST cohomology to the cohomology of the exterior derivative operator $d$ introduced in the previous section.

\section{Negative Ghost Number}

First, let us consider the case with negative ghost number $-p(p>0)$. If $g h(A)=-p$, then one can write,

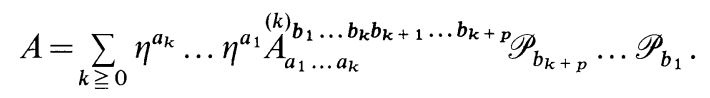

Each term in the sum possesses $p$ more $\mathscr{P}$ 's than $\eta$ 's.

Now, if $A$ is BRST invariant, its zeroth order coefficient

$$
\stackrel{(0)}{A^{b_{1} \ldots b_{p}} \mathscr{P}_{b_{p}} \ldots \mathscr{P}_{b_{1}}}
$$

must obey

$$
\stackrel{(0)}{A}^{a b_{2} \ldots b_{p}} \mathscr{P}_{b_{p}} \ldots \mathscr{P}_{b_{2}} G_{a}=0,
$$

as one easily checks from $[A, \Omega]=0$. This implies, since the constraints are irreducible, that $\stackrel{(0)}{A}$ is $\delta_{2}$-closed, and thus can be written as [8]

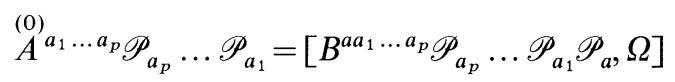

+ terms with at least one $\eta^{a}$, for some function $B^{a a_{1} \ldots a_{p}}$. Hence, the term with no $\eta^{a}$ in $A$ can be removed from $A$ by adding a suitable BRST-exact function $[B, \Omega]$. By the same reasoning, one then shows that the higher order terms $\stackrel{(1)}{A}, \stackrel{(2)}{A} \ldots$ can also be removed, so that one gets

$$
A=[B, \Omega] .
$$

Therefore, the BRST cohomology with negative ghost number is trivial.

\footnotetext{
${ }^{3}$ For a gauge group the $C^{a}{ }_{b c}$ do not depend on phase space. Consequently, in that case the forms $\omega^{a}$ can be detached from the orbits and identified with the Maurer-Cartan form on the gauge group $[13,15]$
} 


\section{Positive Ghost Number}

The cases with $p \geqq 0$ are more interesting. In that instance, each coefficient of $A$ in the expansion in powers of the ghosts contains more $\eta$ 's than $\mathscr{P}$ 's,

$$
A=\sum_{k \geqq 0} \eta^{a_{k+p}} \ldots \eta^{a_{1}} \stackrel{(k)}{A}_{a_{1} \ldots a_{k+p}}^{b_{1} \ldots b_{k}} \mathscr{P}_{b_{k}} \ldots \mathscr{P}_{b_{1}} .
$$

In order to study the implications of BRST invariance for $A$, one needs the first and the second terms in $\Omega$. They are given by $[7,8]$,

$$
\Omega=\eta^{a} G_{a}-\frac{1}{2}(-)^{\varepsilon_{b}} \eta^{b} \eta^{c} C^{a}{ }_{c b} \mathscr{P}_{a}+\text { "more", }
$$

where "more" stands for terms at least quadratic in the ghost momenta. The explicit form of these higher order terms is not needed for the present analysis.

Direct computation of $[A, \Omega]$ shows that the zeroth order term $\stackrel{(0)}{A}$ in the expansion (V.2.1) obeys

$$
(\stackrel{(0)}{d A})_{a_{1} \ldots a_{p+1}} \approx 0
$$

if $[A, \Omega]=0$, since $(d \stackrel{(0)}{A})_{a_{1} \ldots a_{p+1}}$ is, modulo the constraints, the coefficient of the term $\eta^{a_{p+1}} \ldots \eta^{a_{1}}$ in $[A, \Omega]$. It appears therefore natural to interpret the ghosts as 1 -forms along the gauge orbits, that is, to make the identification $\omega^{a}=\eta^{a}{ }^{4}$.

Hence, one concludes from (V.2.3) that BRST invariant functions of positive ghost number $p$ are ghost extensions of closed $p$-forms along the gauge orbits.

One also sees that by adding to $A$ a term $[B, \Omega]$, with

$$
B=\eta^{a_{p-1}} \ldots \eta^{a_{1}{ }_{B} B_{a_{1} \ldots a_{p-1}}^{(0)}}+\eta^{a_{p}} \ldots \eta^{a_{1}} \stackrel{(1)}{B}_{a_{1} \ldots a_{p}}^{b} \mathscr{P}_{b}
$$

the coefficient $\stackrel{(0)}{A}$ in (V.2.1) transforms as

$$
\stackrel{(0)}{A_{a_{1} \ldots a_{p}}} \rightarrow \stackrel{(0)}{A_{a_{1} \ldots a_{p}}}+(\stackrel{(0)}{d B})_{a_{1} \ldots a_{p}}-\stackrel{(1)}{B_{a_{1} \ldots a_{p}}^{b}} G_{b} .
$$

Thus, one can modify the "zeroth order" term $\stackrel{(0)}{A}$ of the ghost number $p$ BRST invariant function $A$ by adding to it an arbitrary exact $p-1$ form along the gauge orbits and an arbitrary combination of the constraints. This means that cohomological classes of $(\operatorname{Ker} \Omega / \operatorname{Im} \Omega)_{\text {class. }}^{p}$ of the BRST generator define, through their zeroth order component, cohomological classes of the weak cohomology introduced in Sect. IV.

In order to complete the demonstration of Theorem 4, we now need to answer two questions: (i) Do all closed $p$-forms $\stackrel{0}{A}, d \stackrel{0}{A} \approx 0$, possess a BRST invariant representative $A$ in the extended phase space? and (ii) Do the higher order components of $A($ i.e., $\stackrel{(1)}{A}, \stackrel{(2)}{A}, \ldots)$ contain any cohomological information?

\footnotetext{
${ }^{4}$ This is reminiscent of what has been done in [13] for the Yang-Mills case
} 
It turns out that the answer to (i) is affirmative, while the answer to (ii) is negative. Therefore, it is indeed true that

$$
\left(\frac{\operatorname{Ker} \Omega}{\operatorname{Im} \Omega}\right)_{\text {classical }}^{p}=\left(\frac{\operatorname{Ker} d}{\operatorname{Im} d}\right)^{p}, \quad p \geqq 0 .
$$

The analysis starts as follows. Consider an arbitrary closed $p$-form

$$
\stackrel{0}{A}=\eta^{a_{1}} \ldots \eta^{a_{p}} \stackrel{(0)}{A_{a_{p} \ldots a_{1}}}, \quad d \stackrel{0}{A} \approx 0 .
$$

The problem is whether one can complete $\stackrel{0}{A}$ by adding terms at least linear in the ghost momenta in such a way that $[A, \Omega]=0$.

Let us write the yet-to-be-found $A$ as in (V.2.1), and evaluate $[A, \Omega]$. The equation $[A, \Omega]=0$ implies equations of the following type for $\stackrel{(k)}{A}$,

$$
(n+1)^{(n+1)} A^{a_{1} \ldots a_{n} a_{n+1}} G_{a_{n+1}}=M^{a_{1} \ldots a_{n}} \quad(n \geqq 0),
$$

where we have set

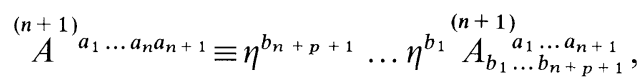

and where $M^{a_{1} \ldots a_{n}}$ stands for a function of $\stackrel{(k)}{A}, k \leqq n$, whose precise form will not be needed here. [For the $p=0$ Hamiltonian, $M^{a_{1} \ldots a_{n}}$ is explicitly written in $[7,8]$.]

Hence, provided that the right-hand sides of (V.2.6) obey

$$
M^{a_{1} \ldots a_{n}} G_{a_{n}}(-)^{\varepsilon_{a_{n}}}=0,
$$

the coefficients $\stackrel{(1)}{A}, \stackrel{(2)}{A}, \ldots$ of $A$ can successively be found from Eqs. (V.2.6) [8].

That the compability conditions (V.2.7) hold, will be proved in a moment. Observe first that the ambiguity in $\stackrel{(n+1)}{A}$ which results from (V.2.6) (for given $\stackrel{(k)}{A}$, $k \leqq n)$, is of the form [8]

$$
\stackrel{(n+1)}{A} a_{1} \ldots a_{n} a_{n+1} \rightarrow \stackrel{(n+1)}{A}^{a_{1} \ldots a_{n} a_{n+1}}+\stackrel{(n+1)}{B} a^{a_{1} \ldots a_{n+1} b} G_{b}(-)^{\varepsilon_{b}} .
$$

This implies that the undetermined term in $\stackrel{(n+1)}{A}$ can be written as

$$
[B, \Omega]+\text { higher order terms in the momenta, }
$$

so that the ambiguity in $A, \stackrel{(0)}{A}$ being given, is completely accounted for by a BRST exact function. Therefore, the zeroth order term $\stackrel{(0)}{A}$ completely determines the BRST cohomological class of $A$, and (ii) above is established.

To show that (V.2.7) holds, one can proceed in two different ways. Either one starts from $d A \approx 0$ and, using repeatedly $d^{2} \approx 0$, one arrives at identities which imply (V.2.7), as done in [8] (these would be the "identities obeyed by the higher 
order structure functions associated with $\stackrel{(0)}{A}$ "). Or one notes, as will be done here, that (V.2.7) is an algebraic condition on $M^{a_{1} \ldots a_{n}}$ which is purely local. To check that the BRST invariant extension of $A$ exists, it is thus not necessary to worry about global questions.

Now, it is well known that the constraints can always be locally "abelianized," i.e., replaced by equivalent constraints which are pure momenta. The analysis of the consistency conditions (V.2.7) is much simpler in that case. Since one goes back to the original constraints by a (local) canonical transformation in the extended phase space, one can assume here that $G_{a}=p_{a}$ for some momenta.

In the case of abelian constraints, $\Omega$ reads

$$
\Omega=\eta^{a} p_{a},
$$

and the condition $[A, \Omega]=0$ implies

$$
(n+1) \stackrel{(n+1)}{A} a \ldots a_{n} a p_{a}=d \stackrel{(n)}{A} a^{a_{1} \ldots a_{n}},
$$

where $d$ is again the exterior derivative along the orbits. For $n=0,(\mathrm{~V} .2 .11)$ can be solved since $d \stackrel{(0)}{A} \approx 0$. For $n>0$, the consistency conditions read

$$
d A^{(n)}{ }^{a_{1} \ldots a_{n}} p_{a_{n}}(-)^{\varepsilon_{a}}=0
$$

and these are clearly fulfilled since

$$
\begin{aligned}
d \stackrel{(n)}{A^{a_{1} \ldots a_{n}}} p_{a_{n}}(-)^{\varepsilon_{a_{n}}} & =d\left(\stackrel{(n)}{A^{a_{1} \ldots a_{n}}} p_{a_{n}}\right) \quad\left(\text { for } d p_{a_{n}}=0\right) \\
& =\frac{1}{n} d^{2\left(\begin{array}{c}
(n-1) \\
A
\end{array} a_{1} \ldots a_{n-1}\right)}
\end{aligned}
$$

[upon use of (V.2.11) for $n$ in place of $n+1$ ]

$$
=0 \text {. }
$$

The last step follows from $d^{2}=0$, which strongly holds for abelian constraints.

Thus, the existence of a BRST invariant extension of ghost number $p$ is proved for any closed $p$-form $\stackrel{(0)}{A}$ in the case of abelian constraints, and also, by the above argument, in the general case of arbitrary first class constraints.

This completes the identification of the BRST cohomology with the cohomology of $d$ for a general dynamical system with a gauge symmetry, when the constraints are irreducible.

\section{Does BRST Cohomology see all the Structure There Is?}

The above theorem relates the BRST cohomology to geometrical objects associated with the structure of the gauge orbits and of the constraint surface. What emerges is that the BRST construction, originally pursued for the sole goal of quantizing gauge systems, possesses interesting topological information about 
the gauge orbits and how they fill the constraint surface. For this reason, we feel that it should constitute an important tool in symplectic geometry. It would be of interest to strengthen our results, by studying the non-irreducible case [22], for which one can also introduce the BRST cohomology. We feel that we have only touched a small part of a much more vast subject.

If the dynamical system is not pure gauge, $(\operatorname{Ker} \Omega / \operatorname{Im} \Omega)_{\text {class. }}^{0}$ does not vanish and contains all gauge invariant functions. In the topologically trivial case, all other cohomological groups vanish. But this is not always so, and it is expected that $(\operatorname{Ker} \Omega / \operatorname{Im} \Omega)_{\text {classical }}^{1}$ is linked with anomalies $[12,13]$ even in the case of an open algebra.

Our analysis also indicates that the group structure can be deemphasized in the BRST construction. It is actually our opinion that it should be deemphasized if one aims at getting a full understanding of the BRST symmetry. Any study in which the group structure plays a prominent role, in spite of its obvious intrinsic interest, might miss the full meaning and generality of BRST methods.

In particular, it might fail to answer the question of what is the geometrical significance, if any, of the higher order structure functions. It should be remarked that the problem still requires further investigation, for our work on the BRST cohomology sheds a poor light on the higher order structure functions. Indeed, only the first two terms in $\Omega$, i.e., only $G_{a}$ and $C_{b c}^{a}$, appear in the definition of the operator $d$, even for an open algebra.

\section{Classical and Quantum Cohomologies Compared and Contrasted}

Finally, it is of interest to compare Theorem 4 to corresponding theorems holding at the quantum level.

The quantum BRST charge is realized as an hermitian operator in some pseudo-Hilbert space on which all dynamical variables, including the ghosts, act as linear operators. We assume that the ordering ambiguity can be resolved in such a way that $\Omega$ is nilpotent,

$$
\Omega^{2}=\frac{1}{2}[\Omega, \Omega]=0 .
$$

In order to implement the condition $\left\langle\psi_{1}|[K, \Omega]| \psi_{2}\right\rangle=0$ for all physical states and any $K$, which expresses that $[K, \Omega]$ is physically unobservable, one defines the physical subspace as

$$
\Omega|\psi\rangle=0,
$$

and the quantum BRST cohomology $\operatorname{Ker} \Omega / \operatorname{Im} \Omega$ by $[16,8]^{5}$

$$
|\psi\rangle \sim|\psi\rangle+\Omega|\chi\rangle
$$

\footnotetext{
${ }^{5}$ It is easy to see that the conditions $\left\langle\psi_{1}|K \Omega+\Omega K| \psi_{2}\right\rangle=0$ for any $K$ (without restriction on the ghost number of $K$ ) implies that the physical space should be included in $\operatorname{Ker} \Omega$. Indeed, let $|\psi\rangle$ $\notin \operatorname{Ker} \Omega$, i.e., $\Omega|\psi\rangle=|t\rangle \neq 0$. Then $|\psi\rangle$ and $|t\rangle$ are linearly independent, since otherwise, the eigenvalues of $\Omega$ would not all vanish, in contraction with $\Omega^{2}=0$. Let $\left|\psi^{\prime}\right\rangle$ be a vector such that $\left\langle\psi \mid \psi^{\prime}\right\rangle \neq 0$. There exists a linear operator $K$ such that $K|t\rangle=\left|\psi^{\prime}\right\rangle$ and $K|\psi\rangle=0(|t\rangle$ and $|\psi\rangle$ are linearly independent). For such a $K$, one has $\langle\psi|K \Omega+\Omega K| \psi\rangle=\left\langle\psi \mid \psi^{\prime}\right\rangle \neq 0$, so that $|\psi\rangle$ cannot be a physical state
} 
The equivalence (V.4.3) follows because $\Omega|\chi\rangle$ is a null state which decouples from all other physical states.

Because $\Omega^{2}=0$, the Jordan form of $\Omega$ involves (in the finite-dimensional case) one-dimensional blocks and two-dimensional blocks. One-dimensional blocks contain states in $\operatorname{Ker} \Omega$ which do not belong to $\operatorname{Im} \Omega$. Two-dimensional blocks are formed by pairs $\left(e_{i}, f_{i}\right)$, with $\Omega f_{i}=e_{i}, \Omega e_{i}=0$. The BRST cohomology removes the two-dimensional blocks. One gets a satisfactory theory if the space of the one dimensional blocks is positive definite - or at least, semi-positive, in which case a further factorization by the left over zero norm states (which are well known to decouple if the space is semi-positive) is needed.

In the Schrödinger representation, one expands the states in powers of the ghosts $\eta^{a}\left(\mathscr{P}_{a}\right.$ becomes $\left.-i \partial / \partial \eta^{a}\right)$

$$
\psi \stackrel{0}{\psi}(q)+\eta^{a} \stackrel{1}{\psi}_{a}(q)+\eta^{b} \eta^{a}{ }^{2} \psi_{a b}(q)+\ldots
$$

The coefficients $\psi^{0}(q), \stackrel{1}{\psi}_{a}(q), \ldots$ are now only functions of the coordinates $q^{i}$ instead of being phase space functions as in the classical expansions (V.1.1, V.2.1).

With the identification of the ghosts with the 1 -forms $\omega^{a}$, the states (V.4.4) can be viewed as generalized forms along the gauge orbits, with coefficients in the space of functions of the coordinates.

The ghost number of a state is equal to its rank as a form, up to an additive constant. This constant can be taken equal to zero (as done in [8]) but the ghost number is then not antihermitian, or equal to $-m^{\prime} / 2+m^{\prime \prime} / 2$, where $m^{\prime}$ is the number of bosonic constraints and $m^{\prime \prime}$ is the number of fermionic constraints. In this last instance, the ghost number operator is antihermitian.

The BRST operator is an operator acting on the forms (V.4.4) which increases the rank by one. Since the constraints are not necessarily linear in the momenta, $\Omega$ is in general a differential operator of order greater than one. This operator appears to be a new geometrical object whose cohomology is hard to compute - or even, to relate to something known.

Things are simpler when the constraints are at most linear in the momenta since then, $\Omega$ can be identified with $e^{S} d e^{-S}$, for some function $S[17,8,18]$ and possesses therefore a clearer geometrical interpretation. The cohomology of $\Omega$ can be linked with more familiar concepts, and an analog of Theorem 4 exists. One sees that if the cohomology of the exterior derivative operator acting on $p$-forms along the gauge orbits with coefficients in the space of functions of the coordinates $q^{i}$ is trivial, then, physical states in the BRST approach only exist at zero ghost number and can be put in correspondence with gauge invariant states $[8,18]$. No extra condition on the ghost number of $|\psi\rangle$ is required. If, however, the topology is nontrivial further conditions might be required to get a sensible theory [17].

[Decoupling theorems are also known for the Fock representation, which is inequivalent to the Schrödinger representation, but these will not be recalled here $[16,18]$.

It appears therefore that the quantum BRST cohomology (V.4.2), (V.4.3) is much harder to characterize than the classical one, except for peculiar dynamical 
systems, for which an analog of Theorem 4 exists and a straightforward geometrical interpretation of $\operatorname{Ker} \Omega / \operatorname{Im} \Omega$ can be given.

\section{Appendix: Exterior Derivative on a Supermanifold: Conventions}

We use conventions such that only one "grading," called the "Grassmann parity," is relevant in the exterior calculus of forms.

The rules which fix these conventions are:

$$
\begin{gathered}
\varepsilon(d F)=\varepsilon_{F}+1, \\
F G=(-)^{\varepsilon_{F} \varepsilon_{G}} G F, \\
d(F G)=F d G+(-)^{\varepsilon_{G}} d F G,
\end{gathered}
$$

where $\varepsilon_{F}$ is the Grassmann parity of the $p$-form $F$ (assumed to be of definite Grassmann parity), and where $F G$ is the exterior product of the forms $F, G$. The Grassmann parity of an even (odd) 0 -form is zero (one).

If, in particular, $z^{A}$ are coordinates of the supermanifold, of Grassmann parity $\varepsilon_{A}$, one finds

$$
\begin{aligned}
z^{A} z^{B} & =(-)^{\varepsilon_{A} \varepsilon_{B}} z^{B} z^{A} \\
z^{A} d z^{B} & =(-)^{\varepsilon_{A}\left(\varepsilon_{B}+1\right)} d z^{B} z^{A} \\
d z^{A} d z^{B} & =(-)^{\left(\varepsilon_{A}+1\right)\left(\varepsilon_{B}+1\right)} d z^{B} d z^{A}
\end{aligned}
$$

These conventions enable one to identify straightforwardly $\omega^{a}$ with the ghosts $\eta^{a}$, since they possess the same algebraic properties. They differ from the conventions of [19], but have been also adopted in [20,21].

Acknowledgements. This work has been supported in part by NSF grant PHY-8600384, by a grant from the Tinker Foundation, and by a NATO Collaborative Research Grant. We are also most grateful to Mike Ratzner for very stimulating conversations.

\section{References}

1. Feynman, R.P.: Quantum theory of gravitation. Acta Phys. Polon. XXIV, 697 (1963)

2. Faddeev, L.D., Popov, V.N.: Feynman diagrams for the Yang-Mills field. Phys. Lett. 25B, 29 (1967)

3. Becchi, C., Rouet, A., Stora, R.: The abelian Higgs Kibble model, unitarity of the $S$-operator. Phys. Lett. 52B, 344 (1974); Renormalization of the abelian Higgs-Kibble model. Commun. Math. Phys. 42, 127 (1975); Ann. Phys. (NY) 98, 287 (1976)

4. Tyutin, I.V.: Gauge invariance in field theory and in statistical mechanics. Lebedev preprint FIAN No. 39, 1975 (unpublished)

5. Dirac, P.A.M.: Lectures on quantum mechanics. Yeshiva University. New York: Academic Press 1964

6. Batalin, I.A., Fradkin, E.S.: Nucl. Phys. B 279, 514 (1987)

7. Batalin, I.A., Vilkovisky, G.A.: Relativistic $S$-matrix of dynamical systems with bosons and fermion constraints. Phys. Lett. 69B, 309 (1977)

Fradkin, E.S., Fradkina, T.E.: Quantization of relativistic systems with boson and fermion first- and second-class constraints. Phys. Lett. 72B, 343 (1978) 
8. Henneaux, M.: Hamiltonian form of the path integral for theories with gauge freedom. Phys. Rep. 126, 1 (1985); see also Structure of constrained Hamiltonian systems and Becchi-RouetStora symmetry. Phys. Rev. Lett. 55, 69 (1985)

9. Batalin, I.A., Fradkin, E.S.: A generalized canonical formalism and quantization of reducible gauge theories. Phys. Lett. 122B, 157 (1983)

10. Tyutin, I.V., Schwartsman, Sh.M.: BRST operator and an open gauge problem. Phys. Lett. 169B, 225 (1986)

11. Batalin, I.A., Fradkin, E.S.: Closing and abelizing operatorial gauge algebra generated by first class constraints. J. Math. Phys. 25, 2426 (1984)

12. Stora, R.: In: New developments in quantum field theories and statistical mechanics. Levy, M., Mitter, P. (eds.). New York: Plenum Press 1977

Baulieu, L.: Cargese Lectures, July 1983. In: Perspectives in particles and fields. Basdevant, J., Levy, M. (eds.). New York: Plenum Press 1985

Baulieu, L.: Perturbative gauge theories. Phys. Rep. 129, 1 (1985)

13. Bonora, L., Cotta-Ramusino, P.: Some remarks on BRS transformations anomalies and the cohomology of the Lie algebra of the group of gauge transformations. Commun. Math. Phys. 87, 589 (1983)

14. Bergmann, P.G.: Observables in general relativity. Rev. Mod. Phys. 33, 510 (1961)

Faddeev, L.D.: The Feynman integral for singular Lagrangians. Theor. Math. Phys. 1, 1 (1969)

15. McMullan, D.: Yang-Mills theory and the Batalin-Fradkin-Vilkovisky formalism. J. Math. Phys. 28, 428 (1987)

Kostant, B., Sternberg, S.: Tel Aviv University preprint 1987

16. Curci, G., Ferrari, R.: An alternate approach to the proof of unitarity for gauge theories. Nuovo Cim. 35A, 273 (1976)

Kugo, T., Ojima, I.: Local covariant operator formalism of non-abelian gauge theories and quark confinement problem. Suppl. Prog. Theor. Phys. No. 66 (1979)

17. Henneaux, M., Teitelboim, C.: In: Quantum field theories and quantum statistics. Batalin, I.A., Isham, C.J., Vilkovisky, G.A. (eds.). Bristol: Adam Hilger 1987 (to appear)

18. Henneaux, M.: BRST symmetry in the classical and quantum theories of gauge systems. In: Proceedings of the Meeting on Quantum Mechanics of Fundamental Systems, at the Centro de Estudios Cientificos de Santiago, CECS Scientific Series. New York: Plenum Press (to appear)

19. Wess, J., Bagger, J.: Supersymmetry and supergravity. Princeton: Princeton University Press 1983

20. Kastler, D., Stora, R.: J. Geom. Phys. 2,1 (1985); Jadczyk, A., Kastler, D.: IHES preprint $\mathrm{P} / 87 / 1$ (Bures-sur-Yvette) 1987

21. Berezin, F.A., Leites, D.A.: Sov. Math. Dok1. 16, 1218 (1975)

Leites, D.A.: Russ. Math. Surv. 35, 1 (1980)

Communicated by L. Alvarez-Gaumé

Received June 5, 1987; in revised form August 8, 1987 